\title{
Protumor immunity and breast cancer development
} LM Coussens

Address: Department of Pathology and Helen Diller Family Comprehensive Cancer Center, University of California, San Francisco, San Francisco, CA 94143, USA

Email: LM Coussens - Lisa.coussens@ucsf.edu

from 6th International Symposium on the Intraductal Approach to Breast Cancer Santa Monica, CA, USA. 19-21 February 2009

Published: 24 July 2009

BMC Proceedings 2009, 3(Suppl 5):SI doi:10.1 186/I753-656I-3-S5-SI

This abstract is available from: http://www.biomedcentral.com/I753-656I/3/S5/SI

(c) 2009 Coussens; licensee BioMed Central Ltd.

For decades, it was generally accepted that leukocytic infiltrations in tumors represented a failed attempt of the immune system to eradicate damaged cells. While indeed some aspects of failed anti-tumor immunity exist, what we now appreciate is the fact that multiple protumor immune programs are instead co-opted by nascent tumors, and in so doing significantly enhance tumor development, including breast cancer. Based upon our evaluation of human clinical specimens revealing significant infiltration of breast tumor tissue by both T lymphocytes and macrophages, we asked the question as to whether adaptive immunity was perhaps enhancing protumor properties of macrophages and thereby potentiating breast carcinogenesis. Utilizing the MMTV-PyMT mouse model of mammary carcinogenesis, $\mathrm{CD} 4^{+} \mathrm{T}$ celldeficient mice, and an ex vivo three-dimensional organoid co-culture model, we revealed a tumor-promoting role for $\mathrm{T}_{\mathrm{H}} 2$-CD4+ $\mathrm{T}$ effector cells that elicit pro-tumor, as opposed to cytotoxic, bioactivities of tumor-associated macrophages (TAMs) and enhancement of pro-metastatic epidermal growth factor (EGF) receptor signaling programs in malignant mammary epithelial cells. These novel findings provide a mechanism explaining how $\mathrm{T}_{\mathrm{H}} 2$-activated TAMs achieve HIGH level expression of EGF necessary for inducing survival, invasive growth and metastatic programs in malignant cells, and together indicate that antitumor acquired immunity, mediated by $\mathrm{CD} 4{ }^{+} \mathrm{T}$ lymphocytes are usurped in pro-tumor microenvironments and instead promote cancer by engaging cellular components of the innate immune system, and identifies new cellular targets, namely $\mathrm{T}_{\mathrm{H}}$ 2-polarized $\mathrm{CD}^{+} \mathrm{T}$ lymphocytes, for anti-cancer therapy.

\section{Acknowledgements}

Research to be presented was supported by grants from the $\mathrm{NIH} / \mathrm{NCl}$ ROICAI30980, ROICAI3256, ROICA098075, P0ICA72006, and a DOD BCRP Era of Hope Scholar Award (W8IXWH-06-I-04I6). 